\title{
INJECTIVE MATRIX FUNCTIONS ${ }^{1}$
}

\author{
BINYAMIN SCHWARZ
}

\begin{abstract}
Univalence of holomorphic (scalar) functions $f(z)$ is generalized to injectivity of holomorphic matrix functions $V(z)=\left(v_{i k}(z)\right)_{1}^{n}$. Local injectivity is characterized by $\left|V^{\prime}\left(z_{0}\right)\right| \neq 0(|A|=\operatorname{det} A)$. The classes $S$ and $\Sigma$ are defined as in the scalar case. For each class a sufficient condition is proved and a necessary condition is conjectured.
\end{abstract}

1. Introduction. Injective vector and matrix functions are defined as follows [4]. Let the (column) vector $v(z)=\left(v_{1}(z), \ldots, v_{n}(z)\right)^{T}$ be holomorphic in a domain $D$ of the $z$-plane (i.e. each component $v_{k}(z)$ is holomorphic in $\left.D\right) . v(z)$ is called injective in $D$, if

$$
v\left(z_{1}\right) \neq v\left(z_{2}\right), \quad z_{1}, z_{2} \in D, z_{1} \neq z_{2} .
$$

An $n \times n$ matrix $V(z)=\left(v_{i k}(z)\right)_{1}^{n}$, holomorphic in $D$, is called injective there, if for every constant vector $c, c \neq 0$, the holomorphic vector function

$$
v(z)=V(z) c
$$

is injective in $D$. The following criterion for matrix injectivity (cf. [4]) will be used throughout this paper. (The determinant of the matrix $A$ is denoted by $|A|$.)

LEMMA 1. The holomorphic matrix $V(z)=\left(v_{i k}(z)\right)_{1}^{n}$ is injective in $D$ if and only if

$$
\left|V\left(z_{1}\right)-V\left(z_{2}\right)\right| \neq 0, \quad z_{1}, z_{2} \in D, z_{1} \neq z_{2} \text {. }
$$

Proof. (3) holds if and only if $\left(V\left(z_{1}\right)-V\left(z_{2}\right)\right) c=0$ implies $c=0$; hence (3) is equivalent to (1), for all $v(z)$ given by (2), $c \neq 0$.

In the scalar case, $n=1,\left|f\left(z_{1}\right)-f\left(z_{2}\right)\right| \neq 0, z_{1}, z_{2} \in D, z_{1} \neq z_{2}$, is the definition of univalence of the holomorphic function $f(z)$ in $D$. Matrix injectivity is thus a generalization of univalence. Sufficient conditions for injectivity of $V(z)$, given by bounds for norms of $V^{\prime \prime}(z) V^{\prime}(z)^{-1}$ and by bounds for norms of the Schwarzian derivative

$$
\{V(z), z\}=\left(V^{\prime \prime}(z) V^{\prime}(z)^{-1}\right)^{\prime}-\frac{1}{2}\left(V^{\prime \prime}(z) V^{\prime}(z)^{-1}\right)^{2},
$$

were found by the use of matrix differential equations [5, Theorems 3.3-3.6, 4.2' and 4.3']. This paper contains some results (and a conjecture) about injectivity, which are not obtained by differential equations.

Received by the editors October 1, 1980.

1980 Mathematics Subject Classification. Primary 30C55; Secondary 15A54.

Key words and phrases. Univalent functions, injective matrix functions.

${ }^{1}$ This research was sponsored by the fund for the Encouragement of Research at the Technion. 


\section{Local injectivity and decidable classes.}

THEOREM 1. Let the matrix $V(z)=\left(v_{i k}(z)\right)_{1}^{n}$ be holomorphic in a domain $D$ and let $V^{\prime}(z)=\left(v_{i k}^{\prime}(z)\right)$ be its derivative. If

$$
\left|V^{\prime}\left(z_{0}\right)\right| \neq 0 \text {, }
$$

$z_{0} \in D$, then $V(z)$ is locally injective at $z_{0}$. Conversely, the condition (5) is also necessary for local injectivity at $z_{0}$, except if $\left|V^{\prime}(z)\right| \equiv 0$ in $D$.

Proof. Let $z_{1}$ and $z_{2}$ be points near $z_{0}$ in $D$. Using

$$
v_{i k}\left(z_{j}\right)=v_{i k}\left(z_{0}\right)+\left(z_{j}-z_{0}\right) v_{i k}^{\prime}\left(z_{0}\right)+\ldots, \quad j=1,2, i, k=1, \ldots, n,
$$

we obtain

$$
V\left(z_{1}\right)-V\left(z_{2}\right)=\left(z_{1}-z_{2}\right) V^{\prime}\left(z_{0}\right)+o\left(\left|z_{1}-z_{0}\right|+\left|z_{2}-z_{0}\right|\right) .
$$

(5) thus implies (3) for $z_{1}$ and $z_{2}$ near $z_{0}, z_{1} \neq z_{2}$.

To prove the necessity ${ }^{2}$ of (5), we assume, by negation $\left|V^{\prime}\left(z_{0}\right)\right|=0$ and $\left|V^{\prime}(z)\right|$ $\not 0$. Let the disk $\left|z-z_{0}\right| \leqslant r$ belong to $D$, and choose $\varepsilon, 0<\varepsilon<r$. Let $\left\{h_{k}\right\}_{1}^{\infty}$ be a sequence of complex numbers such that $0<\left|h_{k}\right|<\varepsilon / 2$ and $\lim _{k \rightarrow \infty} h_{k}=0$. We define the scalar functions

$$
f_{k}(z)=\left|h_{k}^{-1}\left(V\left(z+h_{k}\right)-V(z)\right)\right|, \quad\left|z-z_{0}\right|<\varepsilon / 2,
$$

$k=1,2, \ldots$ Then $\lim _{k \rightarrow \infty} f_{k}(z)=\left|V^{\prime}(z)\right|=f_{0}(z),\left|z-z_{0}\right|<\varepsilon / 2$, and it is easily seen that the convergence is uniform in $\left|z-z_{0}\right|<\varepsilon / 2$. As $f_{0}\left(z_{0}\right)=0, f_{0}(z) \neq 0$, it follows by Hurwitz's theorem that, for large $k, f_{k}(z)$ has at least one zero $z_{k}$ in the above disk; i.e. $\left|V\left(z_{k}+h_{k}\right)-V\left(z_{k}\right)\right|=0$, and $V(z)$ is thus not injective in $\left|z-z_{0}\right|$ $<\varepsilon$. As $\varepsilon, 0<\varepsilon<r$, was arbitrary, this completes the proof.

The following example shows that, if $\left|V^{\prime}(z)\right| \equiv 0, V(z)$ may be injective in the whole plane:

$$
V(z)=\left(\begin{array}{cc}
2 z^{3} & 3 z^{2} \\
3 z^{2} & 6 z
\end{array}\right) ; \quad\left|V^{\prime}(z)\right| \equiv 0
$$

and

$$
\left|V\left(z_{1}\right)-V\left(z_{2}\right)\right|=3\left(z_{1}-z_{2}\right)^{4}
$$

There are some classes of matrix functions $V(z)$ for which it is easy to decide whether $V(z)$ is injective or not. (The eigenvalues of the $n \times n$ matrix $A$ are denoted $\lambda_{j}=\lambda_{j}(A), j=1, \ldots, n$.)

THEOREM 2. (a) A triangular matrix $V(z)=\left(v_{i k}(z)\right)_{1}^{n}$ is injective in a domain $D$ if and only if each diagonal element $v_{k k}(z), k=1, \ldots, n$, is univalent in $D$.

(b) Let $A$ be a constant matrix. The function $V(z)=\exp (z A)$ is injective in $a$ domain $D$ if and only if $|A|=\Pi_{j=1}^{n} \lambda_{j} \neq 0$ and $2 m \pi i / \lambda_{j} \notin D-D, j=1, \ldots, n$, $m=1,2, \ldots$ In particular, $V(z)$ is injective in $|z|<1$ if and only if $0<\left|\lambda_{j}\right|<\pi$, $j=1, \ldots, n[4]$.

${ }^{2}$ I am thankful to Professor D. London for this proof. 
(c) The binomial $P_{m}(z)=z I+z^{m} A_{m}$ is injective in $|z|<1$ if and only if $\left|\lambda_{j}\left(A_{m}\right)\right|$ $\leqslant 1 / m, j=1, \ldots, n$. Similarly $Q_{m}(z)=z I+\left(1 / z^{m}\right) B_{m}$ is injective in $|z|>1$ if and only if $\left|\lambda_{j}\left(B_{m}\right)\right| \leqslant 1 / m, j=1, \ldots, n$.

Proof. (a) This follows directly from Lemma 1: $V\left(z_{1}\right)-V\left(z_{2}\right)$ is also triangular; hence its eigenvalues are the diagonal elements $v_{k k}\left(z_{1}\right)-v_{k k}\left(z_{2}\right), k=1, \ldots, n$.

$$
\left|V\left(z_{1}\right)-V\left(z_{2}\right)\right|=\left|\exp \left(z_{1} A\right)-\exp \left(z_{2} A\right)\right|=\left|\exp \left(z_{1} A\right)\right|\left|I-\exp \left(z_{2}-z_{1}\right) A\right| .
$$

Hence $\left|V\left(z_{1}\right)-V\left(z_{2}\right)\right|=0$ if and only if there exists an eigenvalue such that $\lambda_{j}\left(\exp \left(z_{2}-z_{1}\right) A\right)=1$. As $\lambda_{j}\left(\exp \left(z_{2}-z_{1}\right) A\right)=\exp \left(\left(z_{2}-z_{1}\right) \lambda_{j}(A)\right)$ this happens only if either $\lambda_{j}(A)=0$ or $\left(z_{2}-z_{1}\right) \lambda_{j}(A)= \pm 2 m \pi i, m=1,2, \ldots$

(c) $\left|P_{m}\left(z_{1}\right)-P_{m}\left(z_{2}\right)\right|=\left(z_{1}-z_{2}\right)^{m}\left|I+\left(z_{1}^{m-1}+z_{1}^{m-2} z_{2}+\cdots+z_{2}^{m-1}\right) A_{m}\right|$.

For $\left|z_{1}\right|<1,\left|z_{2}\right|<1, z_{1} \neq z_{2}$, the function $w\left(z_{1}, z_{2}\right)=z_{1}^{m-1}+z_{1}^{m-2} z_{2}$ $+\ldots+z_{2}^{m-1}$ takes all values $w$ in the disk $|w|<m$, and only those. Hence if $\left|\lambda_{j}\left(A_{m}\right)\right|>1 / m$, for a given $j$, then we may find $z_{1}$ and $z_{2}, z_{1} \neq z_{2}$, in $|z|<1$ such that $w\left(z_{1}, z_{2}\right)=-1 / \lambda_{j}\left(A_{m}\right)$ and then $\left|P_{m}\left(z_{1}\right)-P_{m}\left(z_{2}\right)\right|=0$. If $\left|\lambda_{j}\left(A_{m}\right)\right|<1 / m$ for all $j$, then all eigenvalues of $w\left(z_{1}, z_{2}\right) A_{m}$ lie in $|\lambda|<1$ and hence $\left|P_{m}\left(z_{1}\right)-P_{m}\left(z_{2}\right)\right|$ $\neq 0$. A similar proof applies for the second half of (c).

3. The classes $S$ and $\Sigma$. Injectivity is preserved under conformal mappings. If $z=\phi(\zeta)$ maps $\Delta$ onto $D$ and if $V(z)$ is injective in $D$, then $V(\phi(\zeta))=\tilde{V}(\zeta)$ is injective in $\Delta$. For the study of injectivity in simply connected domains we may thus restrict ourselves to the unit disk. Injectivity is also preserved under linear mappings of $V$.

LemMA 2. Let $A, B$ and $C$ be constant $n \times n$ matrices, $|A| \neq 0,|B| \neq 0$. The holomorphic matrix $V(z)=\left(v_{i k}(z)\right)_{1}^{n}$ is injective in $D$ if and only if the same holds for the matrix $\tilde{V}(z)=A V(z) B+C$.

This follows immediately from Lemma 1.

Let $\left\{C_{m}\right\}_{0}^{\infty}$ be a sequence of (constant) $n \times n$ matrices and let $V(z)=C_{0}+z C_{1}$ $+z^{2} C_{2}+\ldots$ be holomorphic in $|z|<1$. We may assume local injectivity; hence, if we disregard the exceptional case $\left|V^{\prime}(z)\right| \equiv 0$, it follows that $\left|V^{\prime}(0)\right|=\left|C_{1}\right| \neq 0$. Instead of the above function it suffices to consider the function $\left(V(z)-C_{0}\right) C_{1}^{-1}$; i.e. we may assume that $V(0)=0$ and $V^{\prime}(0)=I$. In analogy to the scalar case we say that the function

$$
V(z)=z I+z^{2} A_{2}+\ldots
$$

belongs to the class $S$ if $V(z)$ is holomorphic and injective in $|z|<1$. The following generalization of a well-known scalar result [6, p. 212], [3, p. 44] holds.

THEOREM 3. Let $\left\{A_{m}\right\}_{2}^{\infty}$ be a sequence of $n \times n$ matrices and let $\|A\|$ be a given matrix norm. If

$$
\sum_{m=2}^{\infty} m\left\|A_{m}\right\|<1,
$$

then the function $V(z)$, given by (6), belongs to $S$. 
Proof. (7) implies $\varlimsup\left\|A_{m}\right\|^{1 / m} \leqslant 1$; hence $r$, the radius of convergence of the

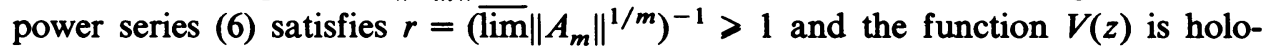
morphic in $|z|<1$. (Note that $r=\min r_{i k}$, where $r_{i k}$ is the radius of the scalar power series $\delta_{i k} z+\sum_{m=2}^{\infty} a_{i k}^{(m)} z^{m}, i, k=1, \ldots, n ; A_{m}=\left(a_{i k}^{(m)}\right)_{1}^{n}, m=2, \ldots$ This follows easily from the existence of positive constants $c_{1}$ and $c_{2}$ such that the inequality

$$
c_{1} \max \left|a_{i k}\right| \leqslant\|A\| \leqslant c_{2} \max \left|a_{i k}\right|
$$

holds for the given norm and any matrix $A=\left(a_{i k}\right)_{1}^{n}[1]$.)

To prove injectivity, let $z_{1}$ and $z_{2}$ be two distinct points in $|z|<1$. By (6),

$$
V\left(z_{1}\right)-V\left(z_{2}\right)=\left(z_{1}-z_{2}\right)\left(I+A\left(z_{1}, z_{2}\right)\right)
$$

where $A\left(z_{1}, z_{2}\right)=\left(z_{1}+z_{2}\right) A_{2}+\left(z_{1}^{2}+z_{1} z_{2}+z_{2}^{2}\right) A_{3}+\ldots$ For all eigenvalues $\lambda_{j}$ $=\lambda_{j}\left(A\left(z_{1}, z_{2}\right)\right), j=1, \ldots, n$, it follows by (7) that

$$
\left|\lambda_{j}\right| \leqslant\left\|A\left(z_{1}, z_{2}\right)\right\|<\sum_{2}^{\infty} m\left\|A_{m}\right\|<1 .
$$

Indeed, we have strict inequality $\left\|A\left(z_{1}, z_{2}\right)\right\|<\Sigma_{2}^{\infty} m\left\|A_{m}\right\|$, except in the case where all $A_{m}=0$. Hence, always $\left|\lambda_{j}\right|<1$ and all the eigenvalues of $I+A\left(z_{1}, z_{2}\right)$ are different from zero. As

$$
\left|V\left(z_{1}\right)-V\left(z_{2}\right)\right|=\left(z_{1}-z_{2}\right)^{n}\left|I+A\left(z_{1}, z_{2}\right)\right|,
$$

this implies $\left|V\left(z_{1}\right)-V\left(z_{2}\right)\right| \neq 0$.

Theorem 3 is sharp. If the matrix norm satisfies $\|I\|=1$, as happens for all induced norms, then no summand $m\left\|A_{m}\right\|$ of assumption (7) can be replaced by $(m-\varepsilon)\left\|A_{m}\right\|, 0<\varepsilon<m$. To show this we note that the function

$$
f_{m}(z)=z-(m-\varepsilon)^{-1} z_{m}
$$

is not univalent in $|z|<1, m=2, \ldots$ Hence $V_{m}=z I-(m-\varepsilon)^{-1} z^{m} I$ is not injective in $|z|<1$ and in this case $(m-\varepsilon)\left\|A_{m}\right\|=1$.

Let $\Sigma$ be the class of functions

$$
W(z)=z I+B_{0}+z^{-1} B_{1}+\ldots
$$

that are holomorphic and injective in $|z|>1$. The following analogue of Theorem 3 holds.

THEOREM 4. Let $\left\{B_{m}\right\}_{0}^{\infty}$ be a sequence of $n \times n$ matrices and let $\|B\|$ be a given matrix norm. If

$$
\sum_{m=1}^{\infty} m\left\|B_{m}\right\|<1
$$

then the function $W(z)$, given by (8), belongs to $\Sigma$.

The proof is similar to the proof of Theorem 3, and the result is again sharp.

Well-known necessary conditions for scalar functions $f(z)=z+a_{2} z^{2}+\ldots$ and $g(z)=z+b_{0}+b_{1} z^{-1}+\ldots$ to belong to the classes $S$ and $\Sigma$ suggest similar necessary conditions in the matrix case. Let $\rho(A)$ denote the spectral radius of the 
$n \times n$ matrix $A$; i.e. $\rho(A)=\max \left|\lambda_{j}(A)\right|$. We conjecture that (a) if $V(z)=z I+z^{2} A_{2}$ $+\ldots$ belongs to $S$, then

$$
\rho\left(A_{2}\right)<2 \text {; }
$$

and (b) if $W(z)=z I+B_{0}+z^{-1} B_{1}+\ldots$ belongs to $\Sigma$, then

$$
\rho\left(B_{1}\right)<1 \text {. }
$$

The following arguments support these conjectures.

(i) The spectral radius $\rho\left(A_{2}\right)\left(\rho\left(B_{1}\right)\right)$ seems to be the appropriate generalization of the absolute value $\left|a_{2}\right|\left(\left|b_{1}\right|\right)$. Indeed the classes $S$ and $\Sigma$ are invariant under similarities induced by constant matrices $T,|T| \neq 0$; if $V(z) \in S$, then also $\tilde{V}(z)=T V(z) T^{-1} \in S$. Such a similarity acts separately on each coefficient; i.e. if $V(z)$ is given by (6) then $\tilde{V}(z)=z I+z^{2} \tilde{A}_{2}+\ldots$, where $\tilde{A}_{2}=T A_{2} T^{-1}$. Hence, $\rho\left(\tilde{A}_{2}\right)=\rho\left(A_{2}\right)$. As norms are not bounded from above under similarities, no necessary condition can be of the form $\left\|A_{2}\right\|<c_{2}\left(\left\|B_{1}\right\|<c_{1}\right)$. E.g., set

$$
V(z)=z I+z^{2}\left(\begin{array}{cc}
1 / 2 & 1 \\
0 & 1 / 2
\end{array}\right)
$$

By Theorem 2(c), $V(z) \in S$. Let $T=\left(\begin{array}{ll}a & 0 \\ 0 & b\end{array}\right), a \neq 0, b \neq 0$; then

$$
\tilde{A}_{2}=T A_{2} T^{-1}=\left(\begin{array}{cc}
1 / 2 & a / b \\
0 & 1 / 2
\end{array}\right)
$$

and $\left\|\tilde{A}_{2}\right\| \rightarrow \infty$ as $(a / b) \rightarrow \infty$.

(ii) The inequalities (10) and (11) hold for all triangular matrices $V(z) \in S$ and $W(z) \in \Sigma$, and are sharp in these cases. This follows, by Theorem 2(a), from the necessity and sharpness of the scalar conditions $\left|a_{2}\right|<2$ and $\left|b_{1}\right|<1$.

(iii) $V(z) \in S$ is similar to a triangular matrix function $\tilde{V}(z) \in S, \tilde{V}(z)=$ $T V(z) T^{-1}$, if and only if all the matrices $\tilde{A}_{m}=T A_{m} T^{-1}$ are triangular. If there exist scalar polynomials $p_{m}(\lambda)$ and a constant matrix $A$, such that $A_{m}=p_{m}(A)$, $m=2, \ldots$, then the matrices $A_{m}$ can be simultaneously transformed into triangular form (say by using $T$ which transforms $A$ into its Jordan form). Hence, for $V(z) \in S$, with $A_{m}=p_{m}(A), m=2, \ldots$, the inequality (10) holds. Similarly, (11) is necessary for an analogous subclass of $\Sigma$.

We remark that conjecture (a) is equivalent to the following conjecture: if $V(z)$ is holomorphic and injective in $|z|<1$, and $\left|V^{\prime}(z)\right| \neq 0$, then

$$
\rho\left(V^{\prime \prime}(z) V^{\prime}(z)^{-1}\right) \leqslant \frac{1+2|z|}{1-|z|^{2}}, \quad|z|<1,
$$

(cf. [3, pp. 20-21]). If the inequality (11) is valid for all $W(z) \in \Sigma$, then the inequality

$$
\rho(\{V(z), z\}) \leqslant \frac{6}{\left(1-|z|^{2}\right)^{2}}, \quad|z|<1,
$$

holds for all functions $V(z)$ which are holomorphic and injective in $|z|<1$, $\left|V^{\prime}(z)\right| \neq 0$ (cf. [2]). We note also that (13) is satisfied in all cases in which the sufficient conditions of Theorems $4.2^{\prime}$ and $4.3^{\prime}$ of [5] hold. 


\section{REFERENCES}

1. P. Lancaster, Theory of matrices, Academic Press, New York, 1969.

2. Z. Nehari, The Schwarzian derivative and schlicht functions, Bull. Amer. Math. Soc. 55 (1949), 545-551.

3. Chr. Pommerenke, Univalent functions, Vandenhoeck and Ruprecht, Göttingen, 1975.

4. B. Schwarz, Injective differential systems, Illinois J. Math. 22 (1978), 610-622.

5. _ Disconjugacy of complex second-order matrix differential systems, J. Analyse Math. 36 (1979), 244-273.

6. E. C. Titchmarsh, The theory of functions, 2nd ed., Oxford Univ. Press, London, 1939.

Department of Mathematics, Technion-Israel Instrtute of Technology, Haifa, IsRael 